\title{
Die telefonische Aufklärung Ein Dauerthema in der täglichen Praxis
}

Viele Ärzte verzweifeln regelmäßig an den Anforderungen, die die Rechtsprechung an die Aufklärung der Patienten stellt. Auch wenn die Aufklärung in der täglichen Arbeit zur Routine gehört, tauchen doch immer wieder dieselben Fragen auf. So zum Beispiel, ob und in welchen Fällen gegebenenfalls eine telefonische Aufklärung erlaubt sein kann.

\begin{abstract}
Telefonische Aufklärung möglich? Bis zu einer Grundsatzentscheidung des Bundesgerichtshofes (BGH) in seinem Urteil vom 15.06.2010 (Az. VI ZR 204/09) galt der eiserne Grundsatz: Aufklärung vor operativen Eingriffen immer nur persönlich im direkten Patientenkontakt! Das Urteil des BGH hat nicht an Brisanz verloren. Neuere Urteile des BGH zu dieser Thematik liegen nicht vor. Dies mag darauf zurückzuführen sein, dass die Urteilsgründe zu Zurückhaltung bei telefonischer Aufklärung mahnen. Nichtsdestotrotz lohnt es sich, sich die Rahmenbedingungen immer wieder einmal zu vergegenwärtigen, um Haftungsrisiken zu minimieren.
\end{abstract}

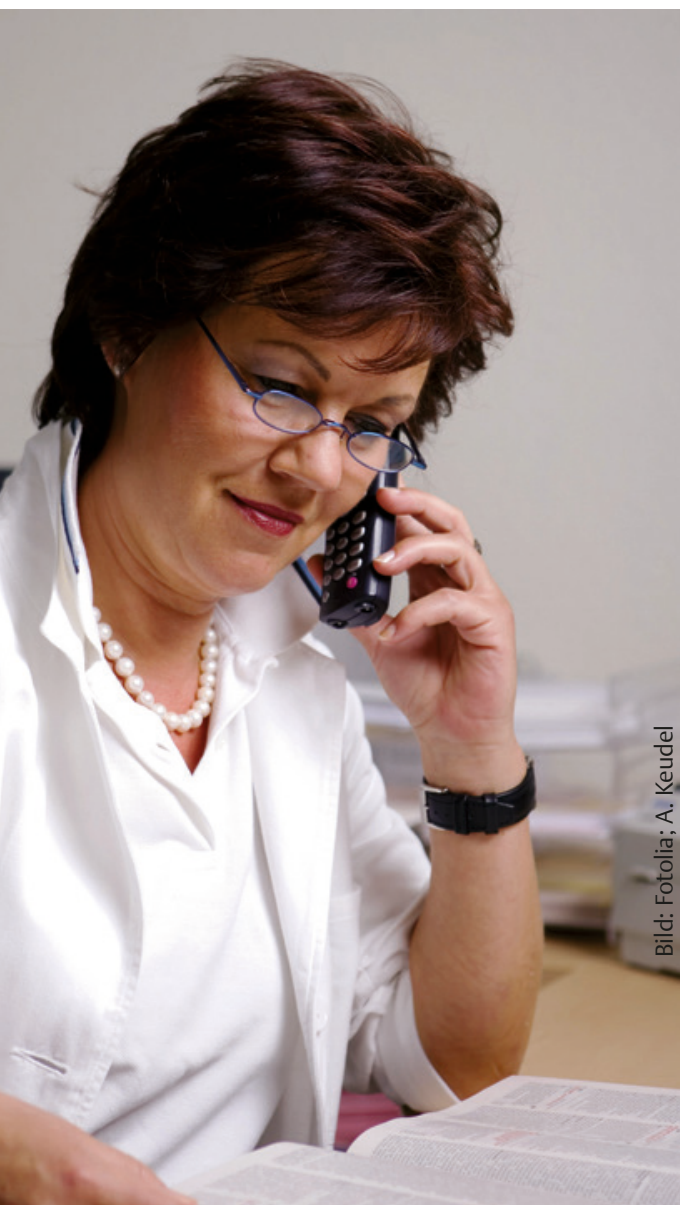

Der Grundsatz der Mündlichkeit der Aufklärung hat auch durch das Patientenrechtegesetz Einzug in das Bürgerliche Gesetzbuch (BGB) gehalten. Dort findet sich in § 630e BGB (Aufklärungspflichten) die Regelung, dass die Aufklärung „mündlich durch den Behandelnden oder durch eine Person erfolgen muss, die über die zur Durchführung der Maßnahme notwendige Ausbildung verfügt; ergänzend kann auch auf Unterlagen Bezug genommen werden, die der Patient in Textform erhält". Hintergrund dafür ist, dass dem Patienten die Möglichkeit gegeben werden soll, dem Behandler gegebenenfalls auch Rückfragen stellen zu können. Der Gesetzeswortlaut schließt dabei aber die telefonische Aufklärung per se nicht aus. Dies bedeutet jedoch nicht, dass eine telefonische Aufklärung in jedem Fall möglich ist. Vielmehr ist hierbei die Rechtsprechung des BGH aus dem Jahr $2010 \mathrm{zu}$ berücksichtigen.

\section{Der Fall}

Verklagt wurden ein Operateur und ein Anästhesist, die bei einem 3 Wochen alten Mädchen eine Leistenhernien-Operation durchgeführt hatten. Der Operateur hatte mit der Mutter der Klägerin in seiner Praxis im Behandlungszimmer ein Aufklärungsgespräch geführt. Die Mutter und der Vater hatten zusätzlich noch ein Aufklärungsformular unterzeichnet. Der Anästhesist hingegen führte 2 Tage vor dem Eingriff mit dem Vater der Klägerin ein 15-minütiges Telefonat über die bevorstehende Operation. Der Inhalt des Gespräches war streitig. Am Morgen der Operation unterzeichneten die Eltern der Klägerin ein Einwilligungsformular. Bei der Operation kam es zu atemwegsbezogenen Komplikationen. Infolge des Narkosezwischenfalls erlitt das Kind eine schwere zentralmotorische Störung. Mit der Klage begehrte die Klägerin Schmerzensgeld und Feststellung einer Schadensersatzpflicht wegen vermeintlicher
Fehler im Zusammenhang mit der Operation. Dabei machte die Klägerin geltend, dass sowohl die chirurgische als auch die anästhesiologische Aufklärung unzureichend gewesen sei, da nicht beide Elternteile aufgeklärt worden seien.

\section{Die Entscheidung}

Der BGH konnte keinen Aufklärungsfehler feststellen.

\section{Grundsatz: beide Eltern müssen einwilligen}

Die Aufklärung des Operateurs sei nicht zu beanstanden gewesen. Die Tatsache, dass nur die Mutter mündlich aufgeklärt wurde, sahen die Richter als unproblematisch an. Zwar bedürfe es nach ständiger Rechtsprechung des erkennenden Senats bei einem minderjährigen Kind in den Fällen, in denen die elterliche Sorge beiden Eltern gemeinsam zusteht, zu einem ärztlichen Heileingriff der Einwilligung beider Elternteile.

\section{Ausnahme Routinefall}

Allerdings könne man jedenfalls in Routinefällen davon ausgehen, dass der mit dem Kind beim Arzt erscheinende Elternteil ermächtigt sei, die Einwilligung in die ärztliche Behandlung für den abwesenden Elternteil mitzuerteilen, worauf der Arzt in Grenzen vertrauen dürfe, solange ihm keine entgegenstehenden Umstände bekannt seien. In anderen Fällen, in denen es um ärztliche Eingriffe schwererer Art mit nicht unbedeutenden Risiken gehe, werde sich der Arzt vergewissern müssen, ob der erschienene Elternteil die Ermächtigung des anderen hat und wie weit diese reicht; er werde aber, solange dem nichts entgegensteht, auf die wahrheitsgemäße Auskunft eines erschienenen Elternteils vertrauen dürfen.

Im konkreten Fall durfte der Operateur nach Meinung der Richter aufgrund der vorliegenden Umstände (relativ einfacher chirurgischer Eingriff, Vater war in 
der Praxis mit anwesend und hat Aufklärungsbogen mit unterzeichnet) davon ausgehen, dass auch der Vater des Kindes einverstanden war. Er durfte insbesondere davon ausgehen, dass der Vater die Mutter ermächtigt hatte, das Aufklärungsgespräch alleine zu führen.

\section{Telefonische Aufklärung nur in einfach gelagerten Fällen}

Im Hinblick auf die anästhesiologische Aufklärung ging der BGH „unter den besonderen Umständen des Streitfalles“ davon aus, dass das Telefongespräch den Anforderungen an ein vertrauensvolles Gespräch zwischen Arzt und Patient entsprach. Der BGH urteilte: „Grundsätzlich kann sich der Arzt in einfach gelagerten Fällen auch in einem telefonischen Aufklärungsgespräch davon überzeugen, dass der Patient die entsprechenden Hinweise und Informationen verstanden hat. Ein Telefongespräch gibt ihm ebenfalls die Möglichkeit, auf individuelle Belange des Patienten einzugehen und eventuelle Fragen zu beantworten (...). Dem Patienten bleibt es unbenommen, auf einem persönlichen Gespräch zu bestehen. Handelt es sich dagegen um komplizierte Eingriffe mit erheblichen Risiken, wird eine telefonische Aufklärung regelmäßig unzureichend sein.“

Im konkreten Fall habe das Aufklärungsgespräch die typischen Risiken einer Anästhesie im Zusammenhang mit einem eher einfachen chirurgischen Eingriff betroffen. Das Telefonat habe 15 Minuten gedauert und sei vom Vater selbst als angenehm und vertrauensvoll bezeichnet worden. Wie die Berufungsinstanz maß auch der BGH dem Umstand besondere Bedeutung bei, dass der Anästhesist bei seinem Telefongespräch mit dem Vater darauf bestanden habe, dass beide Elternteile am Morgen vor der Operation anwesend sind, nochmals Gelegenheit zu Fragen erhalten und sodann ihre Einwilligung zur Operation durch Unterzeichnung des Anästhesiebogens einschließlich der handschriftlichen Vermerke erteilen. Mangels entgegenstehender Anhaltspunkte habe der Anästhesist aufgrund des vorangegangenen telefonischen Aufklärungsgespräches mit dem Vater davon ausgehen dürfen, dass dieser bereits die vorgesehenen ärztlichen Eingriffe und deren Chancen und Risiken mit der Mutter besprochen hatte.

Das Urteil ist jedoch nur mit Vorsicht auf andere Fälle übertragbar. Denn der BGH hat zahlreiche Unwägbarkeiten in das Urteil eingebaut, die in jedem Einzelfall „abgeprüft“ werden müssen.

\section{Einfach gelagerter Fall}

Wesentlicher Punkt im Urteil war, dass es sich um einen einfach gelagerten Fall handelt. $\mathrm{Ob}$ es sich um einen solchen tatsächlich handelt, hatten im konkreten Verfahren Sachverständige zu beurteilen. Im Streitfall liegt das Risiko der Unterscheidung eines einfach gelagerten zu einem schwierigeren Eingriff beim Arzt. Man kann sich vorstellen, dass auch Sachverständige je nach Einzelfall zu unterschiedlichen Ergebnissen kommen könnten.

Folglich sollte sorgsam überlegt werden, ob die telefonische Aufklärung tatsächlich das persönliche Gespräch ersetzen kann oder nicht mehr Probleme als Erleichterung schafft. Nur in völlig unstreitig einfachen, unkomplizierten Eingriffen mit geringen und seltenen Risiken kann die telefonische Aufklärung überhaupt in Erwägung gezogen werden.

Dringend anzuraten ist im Falle einer telefonischen Aufklärung die Dokumentation über

- Datum

- Teilnehmer

- Dauer

- Inhalt (stichwortartig ist ausreichend) des Telefonats sowie

- Gelegenheit des Patienten, Fragen zu stellen (ggf. konkrete Fragen und Antworten dokumentieren).

\section{Einverständnis mit telefonischer Aufklärung}

Der BGH hat deutlich gemacht, dass der Patient mit der telefonischen Aufklärung einverstanden sein muss. Besteht er auf einem persönlichen Gespräch, ist die telefonische Aufklärung ausgeschlossen. Im Optimalfall sollte der Patient vorab schriftlich sein Einverständnis zur telefonischen Aufklärung erteilen. Da dies in den seltensten Fällen praxistauglich sein wird, sollte aber zumindest vor Durchführung des Eingriffs schriftlich die Einwilligung bestätigt werden. Am besten mit dem Zusatz: „Der Patient war mit der telefonischen Aufklärung einverstanden“. Manche Stimmen in der juristischen Literatur gehen sogar so weit, dass eine telefonische Aufklärung nur in Betracht käme, wenn der Patient oder dessen Sorgeberechtigter dem Behandler bekannt sind und er daher sicher weiß, mit wem er telefoniert. Damit soll offensichtlich das Risiko ausgeschlossen werden, dass versehentlich der falsche Adressat aufgeklärt wird und die Aufklärung dann ins Leere ginge. Rechtsprechung hierzu gibt es allerdings soweit ersichtlich bisher nicht.

\section{Vor Eingriff Aufklärungsbogen unterschreiben lassen}

Der BGH hat der Tatsache, dass der Aufklärungsbogen vor der Operation noch unterzeichnet wurde und den Eltern die Gelegenheit eingeräumt wurde, noch Fragen zu stellen, deutliches Gewicht beigemessen.

Hieraus sollte die Schlussfolgerung gezogen werden, dass in jedem Fall vor der Operation noch einmal die Möglichkeit für Fragen eingeräumt (Dokumentation!) und die Einwilligung unterzeichnet wird. Ferner zu beachten ist, dass gemäß § 630 e BGB dem Patienten Abschriften von Unterlagen, die er im Zusammenhang mit der Aufklärung oder Einwilligung unterzeichnet hat, auszuhändigen sind.

\section{Fazit}

Seit dem Urteil des BGH im Jahr 2010 ist eine telefonische Aufklärung zumindest denkbar. Sie kommt jedoch nur in Betracht, wenn der Patient damit einverstanden ist und es sich um einen einfach gelagerten Fall handelt. Für den Arzt stellt sich die Frage, ob die vordergründig vielleicht bequeme telefonische Aufklärung nicht mit zu großen Risiken verbunden ist. Im Falle von Komplikationen trägt er das Risiko des Nachweises, dass der Patient die Aufklärung verstanden hat, ausreichend Fragemöglichkeiten hatte, seine Einwilligung tatsächlich erklärt und es sich um einen einfach gelagerten Fall gehandelt hat. Juristen raten daher nach wie vor zur Zurückhaltung bei der telefonischen Aufklärung.

\section{Korrespondenz}

Dr. iur. Isabel Häser

Rechtsanwältin

ETL Lüdemann Wildfeuer \& Partner

Sonnenstr. 9

80331 München

E-Mail: isabel.haeser@etl.de

www.etl.de 